\title{
The relationship between a spatial gradient for exploratory behavior and latent learning'
}

\author{
R. E. LUBOW ${ }^{2}$ \\ NORTH CAROLINA STATE UNIVERSITY, RALEIGH
}

\begin{abstract}
Magnitude of cul preference changed as a function of time of exploration. Initially the cul close to the start box was most preferred. This preference was retained over a $5 \mathrm{~min}$. period, but its magnitude was decreased. These orderly changes may be useful in explaining one type of latent learning experiment where free exploration precedes acquisition. The acquisition data partially support this position.
\end{abstract}

\section{Introduetion}

It has been demonstrated that, in addition to a temporal gradient of exploratory behavior (e.g., Montgomery, 1952), there also exists a spatial gradient (Lubow, 1959). In general, the frequency of cul entries was a decreasing function of the distance from the start box. This spatial gradient may have implications for latent learning experiments which employ free exploration of a multiple alley maze prior to the introduction of reward (e.g., Tolman \& Honzik, 1930). Typically, the place of reward is that cul which is the farthest from the start box. The probability of a correct response is therefore increased not only as a function of the introduction of reward, but as a function of the previous extinction of the exploratory responses to the spatially closer incorrect culs. Thus, after a free exploration session, if reward were introduced into a cul which was in close proximity to the start box, latent learning should exhibit itself as a decrement. That is, in acquisition, the group which had been pre-exposed to the maze would take more trials to learn to enter the correct cul than a control group which has not been pre-exposed to the maze. On the other hand if after a free exploratory session reward were introduced into a cul which was far from the start box, latent learning would exhibit itself as an increment in performance as compared to a control group which had no free exploration. This type of avoidance learning interacting with the introduction of reward may account for some forms of latent learning.

\section{Method}

The $\mathrm{Ss}$ were 68 albino rats, 34 males and 34 females, ranging in age from 65 to 130 days. Sex and age were distributed equally among the four groups.

The apparatus was a straight alley 48 in long and 4 in wide with four sets of symmetrical culs, 8 in deep and 4 in wide, 8 in from each other. Walls were 5 in high. Walls and floor were made of wood and painted flat gray. The top of the apparatus was covered with $1 / 2$ in wire mesh. A wooden barrier, 1 in high and $1 / 2$ in wide, was placed 1 in from the end of each cul.

All $\mathrm{Ss}$ were placed on $24 \mathrm{hr}$. deprivation for seven days prior to testing. On day eirht the Ss were run approximately $1 \mathrm{hr}$. before feeding. The Ss were divided into four groups. Group 1 had $5 \mathrm{~min}$. free exploration followed by the introduction of reward at cul 1. Group II had $5 \mathrm{~min}$. of free exploration followed by the introduction of reward at cul $3.3^{3}$ Group III had no free exploration and received its reward in cul 1. Group IV received no free exploration and received its reward in cul 3.

On the test day Ss from groups I and II were placed individually into the maze start box and allowed to freely explore the maze for $5 \mathrm{~min}$. At the end of the 5 -min. period $\mathrm{S}$ was removed from the maze and again placed in the start box for the first rewarded acquisition trial. The reward pellet was located at the end of both the correct right and left culs. A trial was scored incorrect when $S$ placed 4 feet in a non-reward cul, and scored correct when $S$ placed 4 feet in a reward cul, reached over the barrier, and consumed the reward pellet.

All $\mathrm{Ss}$ received 10 trials. The order of running was counterbalanced. The intertrial interval was $1 \mathrm{~min}$.

\section{Results and Diseussion}

The relationship between relative frequency of entrance into cul 1 and cul 3 as a function of time during the exploratory period is shown in Fig. 1. These curves are based on the combined data from groups I and II. A comparison of the percentage of total entries into cul 1 with the percentage of total entries into cul 3 during minute 1 shows that cul 1 is entered more frequently than cul 3 ( $p<.005$, Wilcoxon matched-pairs signed-ranks test). Also according to the spatial gradient hypothesis the frequency of entrance to the near cul should decrease as a function of exploration time and the frequency of entrance to the far cul should increase as a function of exploration time. A comparison between the percentage of total entries into cul 1 during minute 1 and during minute 5 indicates a significant decrease ( $p<.001$, Wilcoxen). A similar comparison for cul 3 indicates a significant increase $(p<.001)$. However, cul 1 retains its preference to cul 3 during the fifth minute $(p<.001$, Wilcoxon) .

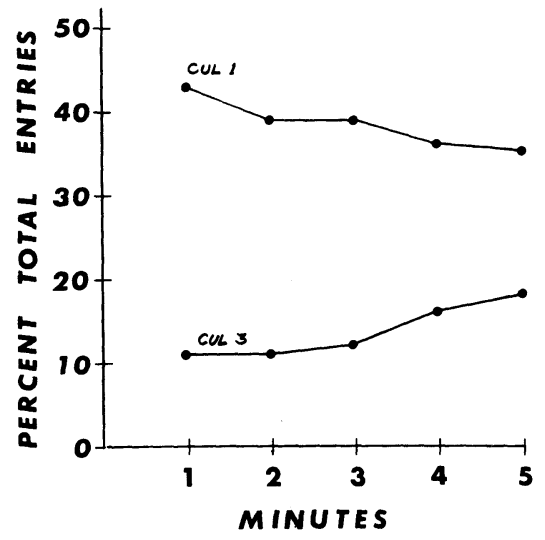

Fig. 1. Percentage of total number of cul entries into cul 1 and into cul 3 as a function of time in exploratory situation. 


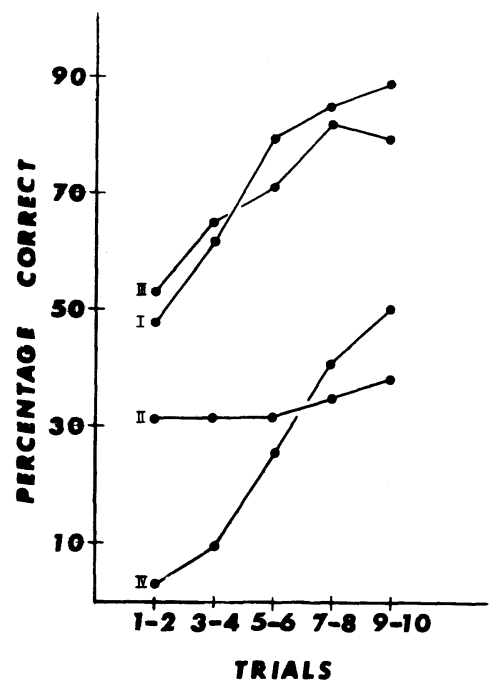

Fig. 2. Percentage correct responses for the four groups as a function of number of trials. Group I, free exploration, reward in cul 1. Group II, free exploration, reward in cul 3. Group III no exploration, reward in cul 2 . Group IV, no exploration, reward in cul 3 .

The course ot learning for each of the four groups over the 10 acquisition trials is shown in Fig. 2. For purposes of analysis the data were divided into two blocks of five trials. For the first block of five trials, Group II (exploratory, reward in cul 3) had significantly more correct responses $(p<.001$, Mann Whitney U-test) than Group IV, (no exploration, reward in cul 3). The differences between groups II and IV for the second block of five trials were not significant. The differences between group I (exploration, reward in cul 1) and group III (no exploration, reward in cul 1) were not significant in the first nor the second blocks of five trials.

It was predicted that the number of correct responses for group II (exploration, reward in cul 3) would be greater than for group IV (no exploration, reward in cul 3). This typical facilitative effect for latent learning was confirmed. However, it was also predicted that group III (no exploration, reward in cul 1) would have a greater number of correct responses than group I (exploration, reward in cul 1). This inhibitory effect of exploration was not confirmed, but neither was there a facilitative effect.
The two predictions were made on the assumption of a characteristic shape of the preceding exploratory gradient. The facilitative effect of the free exploration over no exploration, when reward is subsequently presented in the far cul, is dependent on a decrease in percentage of total entries into the to-be-incorrect culs. The expected results were obtained for the free exploration and learning phases. However, the predicted inhibiting effect of free exploration over no exploration when reward is later presented in cul 1 was not obtained. This occurred in spite of the fact that during free exploration there was the expected decrease with time of total entries into the to-be-correct cul. This can be accounted for by the fact that at the end of the $5 \mathrm{~min}$. exploration period cul 1 was still entered significantly more often than cul 3 , even though cul 1 showed a decrease and cul 3 an increase in preference as a function of time. The inhibiting effect was predicated on this preference being reversed, as a function of free exploration, rather than merely reduced. Longer free exploration with this type of design may allow for obtaining the predicted inhibitory effect.

\section{References}

Lubow, R. E. A spatial gradient for exploratory behavior. Psychol. Rep., 1959, 5, 293-296.

Montgomery, K. C. Exploratory behavior and its relation to spontaneous alternation in a series of maze exposures. $J$. comp. physiol. Psychol., 1952, 45, 50-57.

Tolman, E. C., \& Honzik, C. H. Introduction and removal of reward, and maze performance in rats. Univer. Calif. Publ. Psychol., 1930, 4, 257-275.

\section{Nete.}

1. This research was supported by NIMH Career Development Award K3-MH-7189, Grant MH 08731, and a N. C. State Faculty Research Grant.

2. The author appreciates the assistance of F. Kozoman, C. Williams, and L. Siebert.

3. In the earlier study it was found that the furthermost cul, 4 , had more entries than cul 3 . Once having traversed the length of the maze cul 4 becomes the proximal cul and therefore has an increased probability of entrance. It was thought that this might be obviated by not allowing an extension of the maze beyond cul 4 . However, this blocking of forward movement seems to have even further enhanced the persistance of exploration at that point. Therefore, cul 3 was chosen rather than cul 4. This decision was made on the basis of a prior exploratory study.

\section{Erratum}

Gallup, G. G., Jr. Aggression in rats as a function of nonreward in a straight alley. Psychon. Sci., 1965, $3,99-100$. In the last sentence under "Discussion," it should read as follows: "That conditioned frustration ...." 